\title{
La participación de las víctimas y la sociedad civil en la reformada Corte Africana de Justicia y Derechos Humanos: un estudio comparativo a la luz de la jurisprudencia de la Corte Penal Internacional
}

The participation of victims and civil society in the reformed African Court of Justice and Human Rights. A comparative study in light of ICC's Jurisprudence A participação das vítimas e a sociedade civil na reformada Corte Africana de Justiça e Direitos Humanos: um estudo comparativo à luz da jurisprudência da Corte Penal Internacional

\section{Carmen Montero Ferrer}

Fecha de recepción: 11 de febrero de 2019

Fecha de aprobación: 30 de abril de 2019 Doi: http://dx.doi.org/10.12804/revistas.urosario.edu.co/anidip/a.8503

\begin{abstract}
Para citar: Montero Ferrer, C. (2019). The participation of victims and civil society in the reformed African Court of Justice and Human Rights. A comparative study in light of ICC's Jurisprudence. ANIDIP (7), 141-166. Doi: http://dx.doi.orģ/10.12804/revistas.urosario.edu.co/anidip/a.8503
\end{abstract}

\section{Resumen}

En junio de 2014 la Asamblea de Jefes de Estado y de Gobierno de la Unión Africana adoptó el Protocolo de Enmienda al Estatuto de la Corte Africana de Justicia y Derechos Humanos, conocido como Protocolo de Malabo, que, entre otras cuestiones, crea la Sección de Derecho Internacional Penal (en adelante, SDIP) dotada de jurisdicción para perseguir crímenes de lesa humanidad, genocidio y guerra. En líneas generales,

Investigadora posdoctoral contratada de la Universidad de Santiago de Compostela. Estancia de investigación Université Laval (Canadá) 2018-2020. Becaria de la Xunta de Galicia (Convocatoria de axudas de apoio á etapa de formación posdoutoral nas universidades do SUG, nos oróanismos publicos de investiģación de Galicia. Modalidad A) y miembro del Grupo de Estudios Internacionales de la Universidad de Santiaģo de Compostela (GRESIN-GI-1138). Correo electrónico: carmenmonteroferrer@ǵmail.com. Este trabajo forma parte del proyecto más amplio "Contribuciones de la sociedad civil a la rendición de cuentas por crímenes internacionales. Especial referencia a la Corte Penal Internacional” financiado por dicha convocatoria. 
dicha iniciativa responde a las tensiones surgidas en los últimos años entre algunos estados africanos, la Unión Africana (UA) y la CPI, y a pesar de que hasta la fecha ningún estado ha ratificado el "Protocolo de Malabo", su adopción ha avivado el debate respecto al ejercicio de la justicia internacional penal en África, sobre todo en relación con los conflictos de jurisdicción que podrían ocasionarse entre la SDIP y la CPI, si finalmente dicho instrumento entra en vigor. Nuestro interés está en examinar las posibilidades de participación de las víctimas y la sociedad civil ante la SDIP, en comparación con la práctica seguida por la CPI. Con el propósito de estudiar en qué medida está prevista en el Protocolo de Malabo, y en consecuencia, proponer alternativas que cubran las posibles lagunas, así como valorar si es recomendable que el sistema de participación de la CPI sirva de matriz al desarrollo de esta cuestión en la futura SDIP.

Palabras clave: Protocolo de Malabo, Sección de Derecho Internacional Penal, participación de víctimas, Corte Penal Internacional.

\section{Abstract}

In June 2014 the Assembly of Heads of State and Government of the African Union (AU) meeting in Malabo (Equatorial Guinea) adopted the Protocol to Amend the Statute of the African Court of Justice and Human Rights, known as the Protocol of Malabo; that, among other issues, set up the International Criminal Law Section (ICLS) and its jurisdiction to prosecute crimes against humanity, genocide, and war. The initiative responds to the tensions that have arisen in recent years between some African states, the AU, and the ICC. In despite that no state has ratified the Protocol of Malabo to date, its mere adoption has opened the debate regarding the exercise of international criminal justice in Africa; primarily, due to jurisdictions conflicts that could arise between both courts. The purpose of this paper is to examine the victim's participation before the ICLs regarding the Internal Crime Court (ICC) system and to suggest any ways to make up loopholes. Finally, researchers would like to conclude if the victim's participation system is a procedure the ICLS must follow.

Keywords: Malabo Protocol, International Crimes Law Section, victim's participation, International Criminal Court.

\section{Resumo}

Em junho de 2014 a Assembleia de Chefes de Estado e de Governo da União Africana adotou o Protocolo de Emenda ao Estatuto da Corte Africana de Justiça e Direitos Humanos, conhecido como "Protocolo de Malabo", que, entre outras questões, cria a Seção de Direito Internacional Penal (em adiante, SDIP) dotada de jurisdição 
para perseguir crimes de lesa humanidade, de genocídio e de guerra. Em termos gerais, dita iniciativa responde às tensões surgidas nos últimos anos entre alguns estados africanos, a União Africana (UA) e a CPI, e apesar de que até a data nenhum estado tem ratificado o "Protocolo de Malabo", sua adoção tem avivado o debate respeito ao exercício da justiça internacional penal na África. Especialmente, em relação aos conflitos de jurisdição que poderiam se ocasionar entre a SDIP e a CPI, se finalmente dito instrumento entra em vigor. Nosso interesse está em examinar as possibilidades de participação de vítimas e sociedade civil ante a SDIP, em comparação com a prática seguida pela CPI. Com o propósito de estudar em que medida está prevista no "Protocolo de Malabo", e em consequência, propor alternativas que cubram as possíveis lacunas, assim como valorar se é recomendável que o sistema de participação da CPI sirva de matriz ao desenvolvimento desta questão na futura SDIP.

\section{Palavras-chave: "Protocolo de Malabo", Seção de Direito Internacional Penal, participação de vítimas, Corte Penal Internacional.}

\section{Introducción}

No puede afirmarse que la propuesta de crear un Sistema de Justicia Penal Internacional en África sea del todo novedosa. Durante los debates previos a la adopción de la Carta Africana de Derechos Humanos y de los Pueblos se planteó la posibilidad de crear un órgano jurisdiccional con competencia rationae materiae sobre crímenes de derecho internacional, que finalmente fue rechazada por el Comité de expertos responsable de su elaboración (Abass, 2013). Especialmente, dicha posibilidad parecía quedar descartada a raíz de la creación del Sistema Africano de Protección de Derechos Humanos, de un lado, y el establecimiento de la CPI, de otro.

Sin embargo, la reciente adopción del "Protocolo de Malabo" amenaza dicho reparto competencial al dotar a la Corte Africana de Justicia y Derechos Humanos (CAJDH) de jurisdicción sobre crímenes internacionales y transnacionales, situando a los estados africanos parte del ER ante un sistema de doble complementariedad que podría dar lugar a conflictos de jurisdicción entre la CPI y la SDIP (Mubiala, 2014).

De entre la escasa literatura académica existente, la mayoría de los trabajos publicados hasta ahora discuten la base jurídica sobre la que se asienta la creación de la SDIP, examinando su legitimidad a la luz del principio de complementariedad del ER (Murungu, 2011; Nmehielle, 2014), ${ }^{1}$ o justificando su establecimiento en virtud

1 Sus posturas son contradictorias. Así, Murunģu pone en entredicho la leģitimidad de la SDIP, basándose en el principio de complementariedad (artículos 1 y 17 ER) por considerar que el ER no permite la creación de tribunales reģionales con competencia para juzģar los mismos crímenes que la CPI. Por 
de la obligación de investigar y perseguir los crímenes internacionales recogida en el artículo 4. h) del Acto Constitutivo de la Unión Africana (Mubiala, 2014; Abass, 2013; Ventura y Bleeker, 2016). Por otra parte, existen ciertas voces críticas con la SDIP que enfatizan en la falta de recursos económicos y personales de dicho órgano para asumir el mandato (Mubiala, 2014). Igualmente, son habituales los estudios que argumentan en contra de la inclusión del artículo 46A bis) relativo a la inmunidad de Jefes de Estado y de gobierno durante su mandato (Werle \& Wormbaum, 2017).

Una de las cuestiones menos estudiadas en relación al Protocolo de Malabo es la participación de víctimas y sociedad civil ante la SDIP. Posiblemente, esto sea fruto de la ambigüedad con la que aparece regulada en el citado instrumento, que recuerda a la deficiente e ineficaz redacción del artículo 68.3 ER, hoy superada, tras la adopción de las RPP, y, sobre todo, por la abundante práctica desarrollada por las Salas de la CPI al respecto.

Por esta razón, los objetivos de este trabajo son realizar un análisis prospectivo sobre las opciones que tendrían las víctimas para participar en los procedimientos ante la SDIP, llevar a cabo un análisis crítico de la práctica de la CPI sobre este particular, $y$, en consecuencia, valorar si en un futuro desarrollo normativo del Protocolo de Malabo sobre esta cuestión sería recomendable tomar ejemplo del sistema de la CPI.

Con este fin, seguiremos un método basado en el examen de las decisiones de la CPI sobre participación, y dado que la SDIP no ha entrado en vigor, las fuentes utilizadas en este caso serán, fundamentalmente, el Protocolo de Malabo, otros instrumentos internacionales y regionales, y la doctrina académica existente hasta el momento.

A fin de responder a estas cuestiones, hemos dividido este trabajo en cinco epígrafes. Tras introducir el tema, en el primer epígrafe se abordan los antecedentes y el enfoque actual de la justicia penal internacional en África; así, se trata la cuestión del ámbito jurisdiccional de la CAJDHP y la CPI, y la relación actual de esta última con África. En el segundo, se estudia la interpretación jurisprudencial de la condición de víctima que hace la CPI; además, ante la ausencia de una definición expresa de dicha condición en el Protocolo de Malabo, se analizan las opciones que ofrece el Derecho Internacional para salvar esta laguna. En el tercero, se realiza una revisión crítica del sistema de participación de la CPI y el previsto en el Protocolo de Malabo, centrándonos en los derechos procesales específicos que ejercen las víctimas en cada fase del procedimiento. Finalmente, en el epígrafe cuarto se recogen las conclusiones.

su parte, Nmehielle, citando el trabajo de Murungu, señala que ni el ER ni ningun otro tratado tiene la facultad de reģular en exclusiva ninģuna materia. 


\section{Antecedentes y enfoque actual de la persecución de crímenes internacionales en África}

La adopción del ER y sobre todo el establecimiento de la Corte Penal Internacional fueron especialmente celebrados en África. En un continente resignado a la perpetración impune de crímenes internacionales, la creación de un instrumento, permanente y universal, capaz de llevar a los autores ante la justicia se acogió con entusiasmo. En consecuencia, los estados africanos firmaron y ratificaron masivamente el ER (UA, 2004)², y cuando la CPI inició su actividad, se hicieron partícipes de su mandato colaborando activamente con ella (Soroeta, 2017). ${ }^{3}$

Sin embargo, esta situación ha empezado a cambiar. Desde hace unos años, el buen entendimiento ha dado paso a un creciente malestar entre los dirigentes africanos por la focalización de la actividad judicial de la CPI en África. A lo que se añade el enfado con algunos tribunales europeos por el ejercicio de la jurisdicción universal, al considerar que tales actos vulneran los principios de soberanía e independencia de los estados y evocan el pasado colonialista en África (UA, 2008, Assembly/AU/Dec. 199/XI; AU, 2013, Assembly/AU/Dec.1).

La literatura académica (Graham, 2015; Abass, 2017) coincide en afirmar que la emisión de la orden de arresto contra el presidente sudanés, Omar Hassan Ahmad Al Bashir, dictada por la CPI en marzo de 2009 (Prosecutor vs. Omar Hassan Ahmad Al Bashir, 2009), ${ }^{4}$ fue el detonante que llevó a la UA a adoptar cuatro meses después la Decision on the Meeting of African States Parties to the Rome Statute of the International Criminal Court, que pone de manifiesto su malestar con la CPI y menciona la posibilidad de incluir competencia penal en la Corte Africana de Derechos Humanos y de los Pueblos (UA, 2009, Doc. Assembly/AU/13/XIII).

Fruto de dicho malestar, y al amparo de esta paradigmática "Decisión", Sudáfrica y Gambia anunciaron su intención de retirarse del ER realizando sendas declaraciones oficiales al respecto que, aunque finalmente fueron revocadas, demuestran que la tensión entre África y la CPI continúa latente (Situation in the Republic of Burundi, 2017), ${ }^{5}$ Sin duda alguna, su relación futura dependerá de la capacidad de la CPI de responder ante desafíos como el que le impone la reciente destitución del poder de Al Bashir y, sobre todo, de la voluntad de los estados africanos de ejercer la persecución de crímenes internacionales en sus jurisdicciones nacionales. 
Por otra parte, la entrada en vigor del Protocolo de Malabo añadiría más confusión al panorama de la justicia internacional en África, al ser probable que tuvieran lugar conflictos de jurisdicción entre la CAJDH y la CPI. Aunque seguramente esta situación no se llegue a dar, al menos a corto plazo, ya que al momento de escribir este trabajo ningún país lo había ratificado. Situación que, como señala Bakama Bope, se produce con frecuencia en África, pues es habitual que los instrumentos atraviesen dificultades para ser ratificados (2016). Este es el caso del Protocolo sobre el Estatuto de la Corte Africana de Justicia y Derechos humanos (UA, 2008). Dicho instrumento prevé la creación de la CAJDH, a través de la fusión de la Corte Africana de Derechos Humanos y de los Pueblos con la Corte de Justicia de la Unión Africana; ${ }^{6}$ fue enmendado por el "Protocolo de Malabo", y, aunque se adoptó en 2008, todavía no ha entrado en vigor.

Además, de los estados que han firmado y ratificado o depositado el instrumento de adhesión, sólo nueve han realizado la declaración que reconoce la competencia de la CAJDH para recibir quejas directamente de individuos y ONG, dando muestra de la falta de interés en permitir su acceso a la CAJDH (UA, 2008, artículo 30.f).

En un trabajo publicado con anterioridad a la adopción final del "Protocolo de Malabo", Ademola Abass alegaba que la poca predisposición de los mandatarios africanos a perseguir crímenes internacionales la hacía dudar de que el entonces proyecto de protocolo fuese un valor añadido para poner fin a la impunidad que rodea su comisión (2013). En esta misma línea argumental, consideramos que la escasa voluntad para firmar la declaración que faculta el acceso de individuos y ONG a la CAJDH presagia dificultades para desarrollar un sistema de participación de víctimas y sociedad civil ante la futura SDIP. Bajo esta premisa, surge nuestro interés en estudiar su participación ante dicho órgano jurisdiccional, para lo que empezamos por aproximarnos al concepto de víctima.

\section{Análisis de la condición de víctima en el sistema de la Corte Penal Internacional y la ausencia de definición expresa en el "Protocolo de Malabo"}

En el sistema de la CPI el término víctima se define en la regla 85 RPP. El objeto de dicha regla es concluir quiénes son las víctimas en un caso, de manera que el

6 Al ser el instrumento que prevé la fusión de ambos óroganos jurisdiccionales, éste reemplaza al Protocolo de la Carta Africana para el Establecimiento de una Corte Africana de Derechos Humanos y de los Pueblos (UA, 1998) y al Protocolo de la Corte de Justicia de la Unión Africana (UA, 2003). 
análisis de la condición de víctima constituye el paso previo y conditio sine qua non para su participación en el proceso (Prosecutor vs. Lubanga, 2008a).

Así, como veremos en los siguientes apartados, podrán ser consideradas como victimas las personas naturales o jurídicas que hayan sufrido un daño, dando lugar a uno de los crímenes competencia de la CPI, siempre que exista un vínculo entre el crimen cometido y el perjuicio causado a la víctima.

En virtud de la ausencia en el ER y las RPP de criterios interpretativos que delimiten el alcance y significado de la regla citada, la Sala de Cuestiones preliminares I realizó un primer análisis a propósito del conocido caso Lubanga en la Decision on the Applications for Participation in the Proceedings (Prosecution vs. Lubanga, 2006). Sin embargo, los criterios interpretativos establecidos en este caso no han sido seguidos de manera uniforme por las Salas de Cuestiones Preliminares I y II al aplicar la Regla 85 RPP. Esta cuestión ya ha sido minuciosamente estudiada por la doctrina académica, y por tanto no ha dado lugar en este trabajo (Olásolo \& Kiss, 2010; Vega, 2006).

De este modo, siendo conscientes de que nuestro análisis constituye una aproximación abreviada y generalista sobre este particular, en lo que sigue se van a tratar algunas de las cuestiones que suscitan mayor controversia en la aplicación de la Regla 85 RPP por parte de las Salas de las CPI. Si bien, por la razón expuesta, nos centraremos en el examen de los aspectos más novedosos de la práctica actual.

\section{Personas naturales o jurídicas}

En virtud de la Regla $89.1 \mathrm{RPP}$, en principio sólo aquellas personas que han sufrido un daño a consecuencia de la comisión de uno de los crímenes comprendidos en los artículos 5 a 8 del ER están facultadas para presentar en nombre propio una solicitud al Registro, para que la Sala que corresponda las reconozca como víctimas con el fin de participar en los procedimientos que les afectan. Sin embargo, la interpretación de esta regla ha evolucionado hacia una comprensión más amplia de la misma, y dos situaciones llaman nuestra atención al respecto.

En primer lugar, se ha planteado si una interpretación extensiva de la Regla 85 a) RPP permitiría la participación de las personas fallecidas a través de un pariente que presenta la solicitud en su nombre. Actualmente, las Salas coinciden en no autorizar dicha participación a menos que la víctima hubiese dado su consentimiento antes de fallecer. En ese caso, la participación se limitará a presentar las opiniones y observaciones expresadas por la fallecida en su solicitud inicial (Prosecutor vs. Al Hassan, 2018; Prosecutor vs. Katanga, 2009a; Prosecutor vs. Ntaganda, 2017a). 
En segundo lugar, nos cuestionamos si cabría la presentación conjunta de varias solicitudes con el fin de facilitar la participación de un gran número de víctimas. Parece ser que la tendencia actual va en esta dirección, pues recientemente la CPI ha autorizado solicitudes presentadas por organizaciones no gubernamentales y asociaciones ya existentes con el consentimiento de las víctimas, e incluso por grupos de familias y/o asociaciones constituidas a tal efecto (Prosecutor vs. Al Hassan, 2018).

Por otra parte, la CPI está empezando a desarrollar un sistema de información permanente, destinado a que el Registro y sus unidades especializadas realicen actividades de divulgación sobre el estado de sus investigaciones (Prosecutor vs. Situation in Palestine, 2018; Prosecutor vs. Ongwen, 2015b). Este sistema facilita el contacto con las víctimas y fomenta su participación en los procedimientos (Kiswanson, 2018). Además, podría servir para resolver algunas de las cuestiones que la Asamblea de Estados Partes identifica como dificultades para la participación (ICC-ASP/9/ Res.3, 2010; ICC-ASP/11/Res.8, 2012), como es la necesidad de simplificar y homogeneizar los formularios de solicitud o de clarificar ciertos aspectos confusos sobre la asistencia legal ejercida por los intermediarios (Oficina Pública de Defensa de las Víctimas, 2016).

\section{La teoría del daño}

Dada la poca concreción con la que se define el concepto de daño en la Regla 85 RPP, las salas de la CPI han acudido a la remisión a las normas del Derecho Internacional de los Derechos Humanos que se hace en el artículo 21 ER. Así lo interpretan conforme a los principios 8 y 9 de los Principios y directrices básicos sobre el derecho de las víctimas de violaciones manifiestas de las normas internacionales de derechos humanos $y$ de violaciones graves del Derecho Internacional Humanitario a interponer recursos $y$ obtener reparaciones (Asamblea General de Naciones Unidas, Res.60/147, 2005). Interpretación, con la que la Sala de Apelaciones no se mostró del todo conforme en el caso Lubanga por considerar que los dichos principios son sólo orientativos (Prosecutor vs. Lubanga, 2008a).

Por lo demás, el requisito de conexión entre el daño y el crimen habilita a participar en los procedimientos sólo a las víctimas de los crímenes imputados al acusado en la audiencia de confirmación de cargos (Prosecutor vs. Ntaganda, 2014a; Prosecutor vs. Ntaganda, 2014b). Además, se produce una situación paradójica, pues las víctimas deben formular la solicitud de participación antes de que tenga lugar dicha audiencia. Recientemente, la Sala de Cuestiones Preliminares III ha recurrido al término mutatis mutandis al puntualizar que la víctima debe formular dicha solicitud sobre la base de los actos de los que ha sido víctima y que figuran en la orden de arresto, 
sin perjuicio de que éstos sean o no confirmados en la audiencia de confirmación de cargos (Prosecutor vs. Bemba, 2008).

\section{¿Hacia una admisión automática de la condición de víctima?}

Como ya hemos visto, el análisis sobre la condición de víctima constituye el requisito previo a la participación. La regla 89 RPP define el procedimiento de solicitud sin aclarar si, a estos efectos, la condición de víctima se adquiere de una vez y para siempre, o si por el contrario se habrá de examinar en cada actividad procesal en la que se vaya a participar.

En su interpretación, las salas han diferenciado entre la condición de víctima general y la específica. La primera se examina con anterioridad a la audiencia de confirmación de cargos y autoriza automáticamente a participar en el juicio oral, sin necesidad de readmisión. La segunda define las actividades procesales específicas en las que podrán participar las víctimas sobre la base de la afección de sus intereses personales para el caso concreto (Sthan, Olásolo \& Gibson, 2006). Esta interpretación ha sido recogida en el Manual Práctico de las Cámaras de la CPI por lo que es probable que se mantenga en futuras decisiones de las Salas (2017). ${ }^{7}$

En decisiones recientes, la CPI ha mostrado su intención de dirigirse hacia un sistema de revisión seudoautomática de las solicitudes de participación. Se trata de que el Registro, siguiendo unas pautas fijadas de antemano por las Salas, decida quien serán consideradas como víctimas (Prosecution vs. Ntaganda, 2015; Prosecutor vs. Al Hassan, 2018). Sólo las solicitudes que generen dudas en el Registro serían revisadas por las salas, que se reservan la facultad de supervisión. Este sistema persigue, en consonancia con el mandato de eficacia recogido en la Regla 89. 1 RPP, equilibrar el derecho a participar de las víctimas con los derechos del acusado a ser juzgado sin dilaciones indebidas (ER, 1998, artículo 67.1.c).

\subsection{La ausencia de definición en el Protocolo de Malabo: ¿Cómo suplir esta laguna?}

El Protocolo de Malabo alude en un par de ocasiones a las víctimas sin recoger una defición. En nuestra opinión, esta laguna puede cubrirse a través de dos vías de interpretación del artículo 31 del citado instrumento. La primera consistiría en

7 Conviene aclarar que este no es un instrumento vinculante para los jueces de la CPI, sino que en él se recogen las orientaciones y directrices básicas que les servirán para tomar decisiones con cierta uniformidad de criterio. 
interpretar el precepto en el mismo sentido que hizo la CPI en relación al concepto del daño en el caso Lubanga. De esta manera, proponemos recuperar uno de los argumentos del juez Blattmann para emitir su opinión disidente, pero interpretado en sentido contrario. Es decir, la SDIP acudiría a los "Principios y directrices básicos" para hacer frente a la ausencia de definición (Prosecutor vs. Lubanga, 2012c). ${ }^{8}$

La segunda consistiría en que la SDIP aplicase las resoluciones, decisiones y directivas adoptadas en el seno de la UA. En este sentido podría acudir a la definición de victima recogida en el punto $5 \mathrm{n}$ ) del instrumento Principles and Guidelines on the right to a fair trial and legal assistance in Africa, adoptado por la Comisión de Derechos Humanos y de los Pueblos (ComADHP, 2003) o a la definición recogida en el párrafo 24 del African Union Transitional Justice Framework (UA, 2013).

En conclusión, la interpretación extensiva de la Regla 85 RPP y la búsqueda de mecanismos que facilitan el proceso de solicitud fomentan la participación de víctimas ante la CPI. Por el contrario, la ausencia de definición expresa en el Protocolo de Malabo obstaculiza el ejercicio de sus derechos procesales y pone de manifiesto el escaso interés de los mandatarios africanos en alentar su participación en el proceso penal ante la SDIP, aunque hay definiciones que podrían adoptarse, llegado el caso, para suplir esta laguna.

\section{Revisión crítica sobre participación ante la Corte Penal Internacional y análisis del sistema previsto en el "Protocolo de Malabo"}

En las últimas décadas se ha evolucionado desde una concepción instrumentalista de las víctimas, consideradas como un medio para ejercer la función punitiva de los órganos jurisdiccionales y que sólo actuaban como testigos, a seguir una estrategia orientada por el principio intereses de las víctimas que les permite participar en el proceso penal (Schabas, 2016; Orihuela, 2014). Así, la inclusión de un precepto que regula su participación ante la CPI supuso la consolidación de una nueva manera de entender el papel ejercido por éstas en la justicia penal internacional.

Sin embargo, debido a la escasa concreción con la que fue redactado el artículo 68.3 ER, su aplicación ha dado lugar interpretaciones contradictorias entre las Salas. Cuestión que constituye el objeto de estudio de bastantes trabajos académicos (Sthan, Olásolo \& Gibson, 2006; Vega, 2006; Olásolo \& Kiss, 2010). Razón por la

8 En su opinión disidente y separada, el juez Blattmann considera que, puesto que la RPP ya recogen una definición de víctima en la R.85, es inadecuado e innecesario ir más allá del contenido de esta. 
que, en lo que sigue, centraremos nuestro análisis en aquellos aspectos que suponen cierta novedad respecto a la interpretación que se ha manejado hasta ahora.

Así, a lo largo de los apartados que componen este epígrafe nos proponemos revisar los aspectos más críticos de la participación de víctimas ante la CPI y realizar un examen prospectivo de los derechos procesales que tendrían las víctimas en el caso de que el Protocolo de Malabo entrase en vigor; de esta forma, se va a seguir la estructura del proceso ante la CPI, comparando en cada una de sus fases los derechos procesales previstos en el ER y el "Protocolo de Malabo".

\section{Participación durante la fase de examen preliminar}

\section{El envío de comunicaciones a la Corte Penal Internacional}

Antes de que tenga lugar la confirmación de cargos, los individuos y otros actores de la sociedad civil pueden ejercer una suerte de participación en el proceso al enviar comunicaciones orales y/o escritas a la Fiscalía, que las analizará con el propósito de determinar si existe base razonable para iniciar una investigación de oficio (ER, 1998, artículo 15.1 y 2 ER). Conviene matizar que la Fiscalía ha señalado que el envío de comunicaciones no conduce automáticamente al inicio del examen preliminar de una situación específica (Documento de Política General sobre Exámenes Preliminares, 2013). Si bien, en principio sólo se descartarían las comunicaciones individuales que relaten hechos que no estén dentro de la competencia de la CPI. Cuando las envía una organización, la veracidad de la información se examinará a la luz de su credibilidad y reputación (Prosecutor vs. Situation in the Republic of Côte d'Ivore, 2011). ${ }^{9}$

El estándar de prueba utilizado en esta fase, "base razonable", es el más bajo de todos los previstos en el ER, aunque se valorará con mayor o menor grado de exigencia dependiendo quién haya enviado la información (Piernas López, 2013). Así, si la Fiscalía conoce la notitia criminis por remisión de un Estado parte o del Consejo de Seguridad, estará obligada a iniciar una investigación a menos que determine que no existe fundamento razonable para proceder conforme al Estatuto. Por contra, si recibe una comunicación de parte de individuos u organizaciones de la

9 En este sentido, se pronunció la Fiscalía de la CPI al solicitar autorización a la Sala de Cuestiones Preliminares para abrir una investigación en relación a la situación de Costa de Marfil. Así, la Fiscalía se apoyó en los informes de Human Rights Watch, Aministía Internacional y la Federación Internacional de los Derechos Humanos, elaborados a partir de entrevistas a víctimas y testigos presenciales para acreditar la comisión de crímenes de lesa humanidad (asesinato, violación, otros actos inhumanos y persecución) perpetrados en el contexto de la violencia post-electoral que tuvo luģar en el país africano entre los años 2010 y 2011 . En este caso, la Fiscalía subraya que la credibilidad y los métodos de trabajo de estas orģanizaciones son aro̧umentos suficientes para decidir la apertura de una investigación. 
sociedad civil continuará con la investigación sólo si considera que existe base razonable para proceder (Annex to the Paper on Some Policy Issues Before the Office of the Prosecutor, 2003; Caban, 2011; Schabas, 2010).

\section{El recurso a "otras fuentes fidedignas" como vía para el envío de comunicaciones a la SDIP}

En términos casi idénticos al ER, el Protocolo de Malabo recoge en el artículo 46 G. 2) la posibilidad de que organizaciones como la UA y UN, organizaciones no gubernamentales y "otras fuentes fidedignas" envíen información a la Fiscalía, que constatará su veracidad con el fin de abrir una investigación. Lo que no queda claro es si dentro de éstas últimas entrarían los individuos. En nuestra opinión, la ausencia de mención expresa no debería ser un obstáculo para que los individuos puedan enviar este tipo de comunicaciones, pues el artículo 15 ER tampoco los menciona expresamente, $y$, sin embargo, como hemos visto anteriormente, es una realidad en la actual CPI. (Situation of the Republic of Burundi, 2017).

Finalmente, si se acepta que los individuos envíen comunicaciones, éstos deberán tener derecho, siguiendo el mandato que tiene la Fiscalía de informar, a que se les comunique la decisión de no abrir una investigación (Protocolo de Malabo, 2014, artículo 46.6).

\section{Participación en la fase de confirmación de cargos}

El derecho a presentar opiniones y observaciones se traduce durante la etapa de confirmación de cargos en el derecho a asistir a las audiencias públicas preparatorias, realizar declaraciones de apertura y conclusión a través de sus representantes legales, a ser notificadas de los documentos públicos recogidos en el sumario de las actuaciones, y, si no se trata de víctimas anónimas, a aportar prueba (Prosecutor vs. Katanga, 2008).

\section{La autorización de participación expresa en el Protocolo de Malabo}

Por su parte, las víctimas sí son expresamente autorizadas a presentar observaciones cuando la Fiscalía, tras considerar que existe "fundamento razonable", solicita autorización a la Sala de Cuestiones Preliminares para abrir una investigación formal (Protocolo de Malabo, 2014, artículo 46 G. 3).

\section{Participación en el juicio oral}

El ejercicio de los derechos procesales en los procedimientos ante la CPI a través de los representantes leģales 
La controversia sobre las fases del procedimiento en las que pueden participar las víctimas se deriva del uso del término “juicio” en la versión en español del artículo 68.3 ER, proceedings, en inglés, y procéedure, en francés (Prosecution vs. Lubanga, 2008a). En consecuencia, la CPI ha interpretado el término en sentido estricto para referirse a la fase del juicio oral, poniéndose en entredicho la posibilidad de que las víctimas puedan participar en las fases previas a la apertura de este (Prosecution vs. Lubanga, 2005). Actualmente, prevalece la interpretación extensiva del citado precepto, de tal manera que la jurisprudencia reciente de la CPI confirma su participación desde la fase de examen preliminar (Prosecution vs. Situation in Palestina, 2018).

La controversia surge al tratar de concretar los derechos procesales "caso por caso" (Olásolo \& Kiss, 2010), estrategia que, como señala Bitti, ha dado lugar principalmente durante los primeros años de actividad de la CPI, a notables diferencias en el ejercicio de los mismos (2018). Afortunadamente, en los últimos años se han hecho progresos importantes en este asunto, llegándose a una interpretación más armonizada sobre las actividades específicas que podrán desarrollarse durante esta fase (Prosecutor vs. Ongwen, 2015b). ${ }^{10}$ Actualmente, la jurisprudencia de la CPI establece que las víctimas tienen derecho a ser informadas del trascurso del procedimiento y las actividades procesales que se llevan a cabo, acceder a la documentación pública del caso (Prosecutor vs. Ruto and Sang, 2011), asistir a las sesiones públicas y a puerta cerrada (Prosecutor vs. Katanga, 2010a), hacer declaraciones al principio y al final de la fase del juicio y examinar a los testigos o expertos que presenten prueba (Prosecutor vs. Lubanga, 2010).

Generalmente, las victimas ejercen sus derechos procesales a través de un representante legal. Es importante que se acate el principio básico de las víctimas a elegir a dichos representantes recogido en la Regla 90.1 RPP, ya que éstas se sienten involucradas en el proceso, de modo que se satisface su interés personal (Human Rights Watch, 2017). Además, si las salas lo consideran relevante para conocer la verdad (Prosecutor vs. Katanga, 2010b), las víctimas pueden ejercer sus derechos a título individual, siempre que se respete el derecho de los acusados a ser juzgados sin dilaciones indebidas y a disponer de tiempo suficiente para preparar su defensa (Prosecutor vs. Ntaganda, 2017b; Prosecutor vs. Bemba, 2012).

En nuestra opinión, teniendo en cuenta las dificultades para equilibrar los intereses de ambas partes y siendo además que las víctimas ya pueden satisfacer su derecho

10 Tradicionalmente, las Salas de Cuestiones Preliminares han sido más restrictivas con los derechos procesales de las victimas que las Salas de Primera Instancia, pero esta situación parece que ha empezado a cambiar. 
a "ser oídas" a través del representante legal, la opción de relegar la participación a título individual a aquellas que sean significativas y no puramente simbólicas nos parece recomendable por cuestiones de utilitarismo procesal.

\section{Ausencia de previsión semejante en el Protocolo de Malabo}

El Protocolo de Malabo no prevé expresamente la participación de víctimas durante la fase de juicio oral. La única reseña sobre la comparecencia de las víctimas ante la Corte durante dicha fase la encontramos en el artículo 22 B. 9 a), que trata las medidas de protección. Por otra parte, como hemos visto, se señala explícitamente un derecho de las víctimas a presentar observaciones durante la fase previa al juicio en el artículo 46.G.3). El hecho de que no se establezca un derecho semejante en el citado 22.B 9 a) nos lleva a pensar que cuando la comparecencia se refiere únicamente a la que tiene lugar durante la fase previa al juicio.

\section{Participación en la fase de apelación}

\section{Los límites a la participación en esta fase en los procedimientos de la CPI}

En virtud del artículo $81 \mathrm{ER}$, las víctimas no pueden actuar como apelantes de los pronunciamientos y sentencias dictados por la CPI. Sin embargo, en el caso Lubanga, la Sala de Apelaciones permitió a las víctimas que hubiesen participado durante la fase del juicio y cuyos intereses personales se viesen afectados por la sentencia, presentar sus opiniones y observaciones al respecto a través del representante legal (Prosecutor vs. Lubanga, 2012b). Posteriormente, la sala, a propósito del caso Ngudjolo, ha precisado que dichas opiniones y observaciones deben hacer relación a los errores, de hecho, o de derecho, no presentados por el Fiscal en su apelación, pero que recaigan sobre el ámbito material de la misma (Prosecutor vs. Ngudjolo, 2015). Así, se limita la participación de las víctimas que está intrínsecamente conectada y es dependiente de la actividad judicial de la Fiscalía (Prosecutor vs. Bemba, 2012).

Algo similar ocurre con las decisiones interlocutorias. Las víctimas, al no ser parte procesal, no pueden apelar las decisiones citadas en el artículo 82.1 ER, aunque sí la Orden de reparaciones a través de su representante legal, facultad que se justifica en el hecho de que los intereses personales de las víctimas constituyen la razón de ser de las reparaciones (Prosecutor vs. Bemba, 2012). Por esta misma razón, Bitti considera, a raíz del caso $A l$ Bashir, que la afección de los intereses de las 
víctimas justificaría la apelación de las decisiones interlocutorias sobre competencia o admisibilidad en la fase de examen preliminar (2018). ${ }^{11}$

\section{La falta de regulación de la fase de apelación en el "Protocolo de Malabo"}

Cuando se trata de la participación en la fase de apelación, ninguna conclusión se puede extraer de la lectura del Protocolo de Malabo, ya que, una vez más, se posterga el establecimiento del marco teórico de la propia fase a la adopción de las futuras Reglas de la Corte (Protocolo de Malabo, 2014, artículo 34B).

\section{Participación en la fase de reparaciones}

\section{La inoperatividad del sistema de reparaciones de la CPI}

El artículo 75 ER recoge el derecho de las víctimas a obtener reparaciones, dejando que sea la jurisprudencia la que se pronuncie sobre los principios que serán aplicables a las mismas. Es decir, las Salas de Primera Instancia sólo están obligadas a establecer las orientaciones sobre la base de las cuales el Fondo Fiduciario atenderá las solicitudes individuales (Prosecutor vs. Al Mahdi, 2018). La dependencia económica de dicho órgano, que se nutre de aportaciones estatales, condiciona gravemente su operatividad; además, los condenados suelen ser insolventes. En consecuencia, ambas circunstancias dificultan la operatividad del sistema de reparaciones, impidiendo que éstas sean significativas, apropiadas y adecuadas para las víctimas, y que se otorguen con rapidez, como prescribe el principio 18 de los "Principios y directrices básicos" (AGNU, Res.60/147, 2005).

Por su parte, el inciso tercero del citado artículo 75 recoge el derecho de las víctimas a participar en esta fase, que se ha extendido también a organizaciones no gubernamentales y fundaciones (Prosecutor vs. Al Mahdi, 2016). ${ }^{12}$ La CPI concluye que el hecho de que las víctimas no hayan participado en la fase previa al juicio o durante el mismo no impide que lo hagan en la fase de reparaciones (Prosecutor vs. Lubanga, 2012b; Prosecutor vs. Ongwen, 2015b), si bien su participación debe ser autorizada ex profeso por la Sala (Prosecutor vs. Lubanga, 2015; Prosecutor vs. Katanga, 2017), ${ }^{13}$ lo que no quiere decir que convenga repetir el error cometido en el caso Lubanga en relación a las víctimas de violencia sexual (Prosecutor vs. Lubanga,

\footnotetext{
11 A raíz del caso Al Bashir, en el que se permitió al Reino Hachemita de Jordania apelar una decisión interlocutoria conforme al artículo 82.1.d) ER, Gilbert Bitti considera que no hay precepto leģal que impida a las victimas hacer lo propio.

12 Ambas organizaciones presentaron observaciones en virtud del artículo 75. 3 ER y la Reģla 103 RPP (amicus curiae).

13 En los casos citados la CPI lleģa afirma que, al formular su solicitud de participación, las víctimas relatarán los actos sufridos con el fin de que cualquier examinador considere más verosimil creer que éstas han sufrido el daño declarado, y respecto al cual el acusado ha sido encontrado culpable, que la opción contraria.
} 
2012c). El hecho de que la Sala de Primera Instancia pasase por alto el principio de causalidad constituye un ejemplo de mala praxis que no ha hecho sino fomentar la (re) victimización de las víctimas de dicho caso (Montero Ferrer, 2017). En este sentido, consideramos que no hubiese estado de más la codificación de una serie de principios mínimos que orienten a las víctimas sobre lo que cabe esperar de la fase de reparaciones, en vez de establecerlos "a golpe" de casuística.

\section{La codificación futura de los principios aplicables a las reparaciones en el sistema de la CAJDH}

Posiblemente, el evitar esta mala praxis de la CPI sea la razón que ha llevado a la UA a decidir la codificación de los principios aplicables a las reparaciones (Protocolo de Malabo, 2014, artículo 45.1), a través de la adopción futura de unas "Reglas de la Corte" en las que se descarga, quizá con demasiada ligereza, el desarrollo de todas aquellas cuestiones que la UA no ha tenido a bien recoger en el Protocolo de Malabo.

En suma, como conclusión parcial a este trabajo, reconocemos, tras estudiar la previsión del Protocolo de Malabo sobre participación de víctimas, que no hay lugar a la comparación entre la práctica de la CPI, con una trayectoria de casi dos décadas, y la de la SDIP, que ni siquiera se ha puesto en funcionamiento. Como no podía ser de otro modo, el estudio comparativo sobre los derechos procesales que pueden ejercer las víctimas en ambos órganos queda descompensado en favor de la CPI, como lo presumíamos al iniciar este trabajo, que quisimos abordar con el fin de estudiar en mayor profundidad las afirmaciones que subrayan el impacto negativo que supondría la entrada en vigor del Protocolo de Malabo para el ejercicio del derecho a la participación de víctimas y sociedad civil (Amnistía Internacional, 2016).

\section{Conclusiones}

Antes que nada, conviene precisar que cualquier acción encaminada a poner fin a la impunidad en África merece ser celebrada; la propuesta de crear la SDIP responde a esta idea. Además, el hecho de que recientemente la UA y algunos de sus Estados parte hayan puesto en entredicho la persecución de crímenes internacionales que ejerce la CPI, pone de manifiesto que está surgiendo cierto interés en el continente por el desarrollo de la justicia penal regional. Sucede, sin embargo, que la voluntad política por convertir dicho interés en realidad no es suficiente, como atestigua la falta de ratificación del Protocolo de Malabo.

En esta misma línea argumental, la retirada de algunos estados del ER abriría la vía a la impunidad, esencialmente en países que no cuentan con legislación sobre 
crímenes internacionales, no tienen voluntad de investigar y/o perseguir en su sistema judicial o no disponen de los recursos económicos y materiales para hacerlo.

Ante este panorama, las víctimas siguen siendo las grandes perjudicadas. Para ellas, la ausencia de procedimientos penales en los ámbitos internacional, regional o nacional se traduce en impunidad y olvido. Simplemente por esta razón es que consideramos que las víctimas deberían centrar el debate sobre la CPI y la SDIP. El Derecho Internacional Penal actual reconoce el derecho de las víctimas a un recurso efectivo ante los tribunales y organismos internacionales, y según autores como Orihuela (2014) sirve de fundamento para su participación en los procedimientos de la CPI. Por esta razón, entendemos que la discusión sobre la SDIP debería incluir el análisis de su capacidad para satisfacer este derecho.

En este sentido, resulta evidente que el Protocolo de Malabo ofrece escasas garantías de participación a las víctimas y a la sociedad civil ante la SDIP. Consideramos que el problema no está en la escasa regulación de la participación en el Protocolo de Malabo, pues lo mismo ocurre con el ER respecto del artículo 68.3 ER. Tampoco parece determinante el hecho de que se postergue la regulación de aspectos clave a la adopción futura de las Reglas de la Corte. En nuestra opinión, el verdadero obstáculo para la participación estaría en la falta de interés de los mandatarios africanos en esta cuestión, que se refleja en las dificultades del Protocolo de Estatuto de la Corte Africana de Justicia y Derechos Humanos y el "Protocolo de Malabo" para ser ratificados y/o entrar en vigor, y, sobre todo, en la escasez de declaraciones realizadas por los estados permitiendo el acceso de las víctimas y sociedad civil a la CAJDH.

Por otra parte, se ha de resaltar la evolución de las Salas de la CPI hacia una interpretación cada vez más amplia de preceptos del ER o las RPP que regulan la participación. Cabe destacar la aplicación extensiva de la Regla 89, que está permitiendo la presentación conjunta de solicitudes de participación. También la creación de un sistema permanente de información que, cumpliendo con el mandato de información de la Regla 92 RPP, pone en marcha actividades de divulgación y sensibilización con el propósito de informar a las víctimas sobre el alcance y significado de su derecho a participar en los procedimientos de la CPI.

En nuestra opinión, ambas iniciativas responden a la asunción por parte de la CPI de la tendencia actual que busca fomentar la participación de víctimas y sociedad civil en la persecución de crímenes internacionales, tanto en el plano nacional como internacional, es decir, parece como si la CPI estuviese tomando nota de las críticas que señalan la invisibilidad de las víctimas en el proceso de la CPI, que contrasta con el protagonismo que tienen éstas en los procedimientos por crímenes internacionales seguidos en instancias nacionales (Brody, 2017; Roth-Arriaza, 2005). Por 
su parte es especialmente relevante el caso Hissène Habré, en el que las víctimas, apoyadas por una coalición de organizaciones y asociaciones sin ánimo de lucro, dieron el impulso definitivo al proceso penal seguido contra el ex dictador del Chad en las Cámaras Africanas Extraordinarias en Senegal (CAE), que concluyó con la culpabilidad del acusado por la comisión de crímenes contra la humanidad y la condena a prisión permanente en 2016.

De este modo, convendría que la SDIP, además de reproducir las buenas prácticas de la CPI en cuestiones como la interpretación extensiva de la condición de víctima, siguiese el buen ejemplo de las CAE en ciertos aspectos relativos a la participación. En particular, la SDIP debería tomar nota de los derechos procesales ejercidos por las víctimas durante el juicio oral, en el que 69 víctimas prestaron declaración, y su participación en la fase de reparaciones, en la que éstas pudieron apelar la orden de compensaciones (Brody, 2017; Cruvellier, 2016).

Por todo ello, queremos concluir este trabajo volviendo al punto de partida en la persecución de crímenes internacionales; de esta manera, consideramos que a corto plazo el derecho de las víctimas africanas a participar en los procedimientos penales se habrá de satisfacer a través del ejercicio de la jurisdicción universal. Como hemos visto, el caso Hissène Habré ha iniciado el camino en esta dirección, contrariando las opiniones de aquellos que, con buen criterio, dadas las circunstancias del momento, consideraron hace poco más de un lustro que el ejercicio de la justicia penal internacional en África era un imposible (Abass, 2013). Ante esta situación, la CPI, y, también la UA, tienen un importante papel que desempeñar, pues en virtud de la complementariedad positiva podrán asistir a los estados africanos en el ejercicio de la investigación y persecución de crímenes internacionales.

\section{Referencias}

Abass, A. (2013). Prosecuting International Crimes in Africa: Rationale, Prospects and Challenges. European Journal of International Law, 24 (3), 933-946.

Abass, A. (2017). Historical and Political Background to the Malabo Protocol. En G. Werle y M. Varmbaum (eds.), The African Criminal Court. A Commentary on the Malabo Protocol (pp. 11-28). La Haya: Asser Press y Springer.

Bakama Bope, E. (2017). La nouvelle section pénale de la Cour africaine des droits de l'homme et des peuples: un miroir aux alouettes? En H.J. van der Merwel y G. Kemp (eds.), International Criminal Justice in Africa (pp. 129-165). Nairobi: Konrad Adenauer Stiftung. Strathore University Press. 
Bitti, G. (2018). A Court for Victims? En Federación Internacional de Derechos Humanos (eds.), Victims at the Center of Justice. From 1998 to 2018: Reflections on the promises and the Promises and the Reality of Victim Participation at the ICC (pp.1-15). Paris: Federación Internacional por los Derechos Humanos. Disponible en: www.fidh.org

Brody, R. (2017). Victims bring a Dictator to Justice. The Case of Hissène Habré. Analysis 70. Berlin: Brot für die Welt.

Cavan, P. (2011). Preliminary Examinations by the Office of the Prosecutor of the International Criminal Court. Czech Yearbook of Public \& Private International Law, 2,199-216.

Lopez Piernas, J. J. (2013). Estudio sobre la práctica de la Corte Penal Internacional en materia de investigaciones preliminares a la luz de la reciente remisión de la Unión de Comores. Anuario Español de Derecho Internacional, 29, 327-361.

Montero Ferrer, C. (2017). Crímenes internacionales de violencia sexual e impunidad: un examen de los mecanismos de justicia transicional y su aplicación en África (Tesis doctoral). Universidad de Santiago de Compostela, Santiago de Compostela, España.

Mubiala, M. (2014). Cronique de droit pénal de l'Union Africaine: Lélargissement du mandat de la Cour africaine de Justice et des droits de l'homme aux affaires de droit international pénal. Revue internationale de droit pénal, 85(4), 749-758.

Murungu, C. T. (2011). Towards a Criminal Chamber in the African Court of Justice and Human Rights. Journal of International Criminal Justice, 9(5), 1067-1088.

Nmehielle, V. (2014). Saddling the new African regional human rights courts with international criminal jurisdiction: Innovative, Obstructive, Expedient? African Journal of Legal Studies, 7(1), 7-42.

García San José, D. (2000). La configuración jurídica de las víctimas de los crímenes de la competencia de la Corte Penal Internacional. En J. A. Carrillo Salcedo (ed.), La criminalización de la barbarie: la Corte Penal Internacional (pp. 457-479). Madrid: Consejo General del Poder Judicial.

Graham, G. (2015). Africa's Evolving Continental Court Structures: At the Crossroads? Occasional Paper 209. Governance and APRM Programme. South Africa: South African Institute of International Affairs (SAIIA). 
Kiswanson, N. (2018). Beyond Victim Participation during Proceedings: Outreach and Information Activities during Preliminary Examination in Palestine. En Federación Internacional de Derechos Humanos (eds.), Victims at the Center of Justice From 1998 to 2018: Reflections on the Promises and the Reality of Victim Participation at the ICC, (pp. 43-47). París. Federación Internacional por los Derechos Humanos. Disponible en: www.fidh.org

Olásolo, H., \& Kiss, A. (2010). El Estatuto de Roma y la jurisprudencia de la Corte Penal Internacional en materia de participación de víctimas. Revista Electrónica de Ciencia Penal y Criminología, 12-13, 1-37.

Orihuela Calatayud, E. (2014). Las víctimas de la Corte Penal Internacional. Análisis de la participación de víctimas en la Corte. Pamplona: Thomson Reuters, Aranzadi.

Roth-Arriaza, N. (2005). The Pinochet Effect: Transnational Justice in the Age of Human Rights. Philadelphia: University of Pennsylvania Press.

Schabas, W.A. (2016). The International Criminal Court. A Commentary on the Rome Statute. Oxford: Oxford University Press.

Soroeta, J. (2017). La CPI (2002-2017): Balance y perspectivas, Documento de Investigación 19/2017. Programa de "Trabajo de Futuros"/"Panorama de tendencias geopolíticas. Madrid: Instituto Español de Estudios Estratégicos.

Stahn, C., Olasolo, H., \& Gibson, K. (2006). Participation of Victims in Pre-Trial Proceedings of the ICC, Journal of International Criminal Justice 4, 219-238.

Vega González, P. (2006). El papel de las víctimas en los procedimientos ante la Corte Penal Internacional: sus derechos y las principales decisiones de la Corte, Revista Internacional de Derechos Humanos, 5 (3), 19-41.

Ventura, M.J y Bleeker, A.J (2016). Universal Jurisdiction, African Perceptions of the International Criminal Court and the New AU Protocol on Amendments to the Protocol on the Statute of the African Court of Justice and Human Rights. En E. A. Ankumah (eds.), The International Criminal Court. One Decade On (pp. 441-456). Cambridge: Intersentia \& Africa Legal Aid.

Werle, G., \& Vormbaum, M. (2017). The African Criminal Court: A Commentary on the Malabo Protocol. Berlín: Springer y Asser Press. 


\section{Tratados internacionales y otros instrumentos internacionales}

Asamblea de Estados Partes. (1998). Estatuto de Roma. 17 de julio.

Asamblea de Estados Partes. (2010). Strengfthening the ICC and the Assembly of States parties, 10 de diciembre (Resolution ICC-ASP/9/Res.3).

Asamblea de Estados Partes. (2012). Strengthening the ICC and the Assembly of States Parties, 21 de noviembre (ICC-ASP/11/Res.8).

Asamblea General de Naciones Unidas (2005). Principios y directrices básicos sobre el derecho de las víctimas de violaciones manifiestas de las normas internacionales de derechos humanos y de las violaciones graves del derecho internacional humanitario a inteponer recursos y obtener reparaciones. $16 \mathrm{de}$ diciembre (Res.60/147).

Corte Penal Internacional. (2017). Chambers Practice Manual.

Fiscalía de la Corte Penal Internacional. (2003). Annex to the Paper on some policy issues before the Office of the Prosecutor.

Fiscalía de la Corte Penal Internacional. (2013). Documento de Política General sobre Exámenes Preliminares. 2 de noviembre.

Fiscalía de la Corte Penal Internacional. (2017). Report on Preliminary Examinations Activities 2017. 4 de diciembre.

Oficina Pública de Defensa de las Víctimas. (2013). Representación de víctimas ante la Corte Penal Internacional. Manual para los Representantes legales.

Organización para la Unidad Africana. (1981). Carta Africana de Derechos Humanos y de los Pueblos. 27 de julio de 1981.

Unión Africana. (2000). Acto Constitutivo de la Unión Africana. 7 de noviembre.

Unión Africana. (1998). Protocolo a la Carta Africana para el Establecimiento de una Corte Africana de Derechos Humanos y de los Pueblos.

Unión Africana. (2003). Protocolo de la Corte de Justicia de la Unión Africana. 11 de julio.

Unión Africana. (2008). Protocolo sobre el Estatuto de la Corte Africana de Justicia y Derechos Humanos.

Unión Africana (2014). Protocolo de Enmienda del Estatuto de la Corte Africana de Justicia y Derechos Humanos ("Protocolo de Malabo"). 23 de junio de 2014. 
Unión Africana. (2009). Decision on the Report of the Commission on the Abuse of the Principle of Universal Jurisdiction. 1-3 de julio (Doc. Assembly/AU/ Dec. 199/XI).

Unión Africana. (2009). Decision on the Meeting of African States Parties to the Rome Statute of the International Criminal Court. 1-3 de julio de 2009 (Doc. Assembly/AU/13/XIII).

Unión Africana. (2013). Decision on Africa’s Relationship with the International Criminal Court. 12 de octubre (Doc. No. Ext/Assembly/AU/Dec.1).

Unión Africana, Comisión Africana de Derechos Humanos y de los Pueblos. (2004). Plan Estratégico de la Comisión de la Unión Africana. Volume 3: 2004-2007. mayo de 2004.

Unión Africana, Comisión Africana de Derechos Humanos y de los Pueblos. (2003). Principles and Guidelines on the Right to a Fair Trial and Legal assistance in Africa (Doc/OS (XXX)247).

Unión Africana. (2013). African Union Transitional Justice Framework.

\section{Jurisprudencia}

CPI, Sala de Cuestiones Preliminares I. (2018). Prosecutor vs. Al Hassan Ag Abdoul Aziz Ag. Mohamed Ag. Mahmoud. Decision establishing the principles applicable to victims' applications for participation. 24 de mayo (ICC-01/12-01/18).

CPI, Sala de Primera Instancia III. (2016). Prosecutor vs. Al Mahdi. Observations conjointes de la FIDH et de l'AMDH sur la procédure de réparations. 2 de diciembre (ICC-/01/12-01/15).

CPI, Sala de Apelaciones. (2018). Prosecutor vs. Al Mahdi. Public Redacted Judgment on the appeal of the victims against the "Reparations Order. 8 de marzo (ICC01/12-01/15-259-Red2).

CPI, Sala de Cuestiones Preliminares III. (2008). Prosecutor vs. Jean-Pierre Bemba Gombo. Fourth Decision on Victim's Participation. 15 de diciembre (ICC01/05-01/08-320).

CPI, Sala de Primera Instancia III. (2012). Prosecutor vs. Jean-Pierre Bemba Gombo. Decision on the supplemented applications by the legal representatives of victims to present evidence and the views and concerns of victims. $22 \mathrm{de}$ febrero (ICC-01/05-01/08-2138). 
CPI, Sala de Cuestiones Preliminares III. (2017). Situation in the Republic of Burundi. Decision Pursuant to Article 15 of the Rome Statute on the Authorization of an Investigation into the Situation in the Republic of Burundi. 25 de octubre (ICC-01/17-X-9-US-Exp).

CPI, Sala de Cuestiones Preliminares III. (2011). Situation in the Republic of Côte d' Ivore. Request for authorisation of an investigation pursuant to article 15.23 de junio (ICC-02/11-3).

CPI, Sala de Cuestiones Preliminares I. (2008a). Prosecutor vs. Katanga. Decision on the 97 Applications for Participation at the Pre-Trial Stage of the Case. 10 de junio (ICC- 01/04-01/07-579).

CPI, Sala de Cuestiones Preliminares I. (2008b). Prosecutor vs. Katanga. Decision on the Set of Procedural Rights Attached to Procedural Status of Victim at the Pre-Trial Stage of the Case. 15 de mayo (ICC-01/04-01/07).

CPI, Sala de Primera Instancia II. (2009). Prosecutor vs. Katanga. Grounds for the Decision on the 345 Applications for Participation in the Proceedings Submitted by Victims. 23 de septiembre (ICC-01/04-01/07-1483-Red2).

CPI, Sala de Primera Instancia II. (2010a). Prosecutor vs. Katanga. Decision on the Modalities of Victim Participation at Trial. 20 de enero (ICC-01/04-01/071788-Teng)

CPI, Sala de Apelaciones. (2010b). Prosecutor vs. Katanga. Judgement on the Appeal of Mr. Katanga Against the Decision of Trial Chamber of January 2010 Entitled 'Decision on the Modalities of Victim Participation at Trial'. 16 de julio (ICC-01/04-01/07-2288).

CPI, Sala de Primera Instancia II. (2017). Prosecutor vs. Katanga. Order for reparations pursuant to Article 75 of the Statute. 24 de marzo (ICC-01/0401/07-3758-Teng).

CPI, Sala de Cuestiones Preliminares II. (2014a). Prosecutor vs. Ntaganda. Decision on victims'participation in trial proceedings. 15 de enero (ICC-01/04-02/06-211).

CPI, Sala de Cuestiones Preliminares II. (2014b). Prosecutor vs. Ntaganda. Second Decision on victims' participation at the confirmation of charges hearing and in the related proceedings. 7 de febrero. (ICC-01/04-02/06-251).

CPI, Sala de Primera Instancia VI. (2015). Prosecutor vs. Ntaganda. Decision on victims' participation in trial proceedings. 6 de febero (ICC-01/04-02/06-449). 
CPI, Sala de Primera Instancia VI. (2017). Prosecutor vs. Ntaganda. Decision on 'Ninth Decision on victims'participation in trial proceedings'. 23 de noviembre (ICC-01/04-02/06-2126).

CPI, Sala de Primera Instancia VI. (2017b). Prosecutor vs. Ntaganda. Public redacted version of 'Decision on the request of the legal representation of victims of the attacks for leave to presente evidence and victim's views and concerns'. 15 de febrero (ICC-01/04-02/06-1739-Red2).

CPI, Sala de Apelaciones. (2015). Prosecutor vs. Ngudjolo. Judgment on the Prosecutor's Appeal against the 'Judgment pursuant to Article 74 of the Statute'. 27 de febrero (ICC-01/04-02/12-271-Corr).

CPI, Fiscalía. (2005). Prosecutor vs. Thomas Lubanga Dylo. Prosecution's Reply on the Applications for Participation 01/04-1-dp to 01/04-6-dp. 15 de agosto (ICC-01/04-84).

CPI, Sala de Cuestiones Preliminares. (2007). Proseuctor vs. Thomas Lubanga Dylo. Decision on the Applications for Participation in the Proceedings of VRS 1,VRS 2, VRS 3, VRS 4, VRS5 and VRS 6. 17 de enero (ICC-01/04-101-tEN-Corr).

CPI, Sala de Primera Instancia I (2008a). Prosecutor v. Thomas Lubanga Dylo. Decision on victims'participation. 21 de enero (ICC-01/04-01/06-1119).

CPI, Sala de Apelaciones. (2008b). Prosecutor v. Thomas Lubanga Dylo. Judgement on the appeals of the Prosecutor and the Defence against Trial Chamber I's Decision on victims participation of 18 january 2008. 11 de julio (ICC01/04-01/06-1432).

CPI, Sala de Primera Instancia I. (2010). Prosecutor vs. Thomas Lubanga Dylo. Decision on the Defence Observations regarding the Right of the Legal Representatives of Victims to Question Defence Witness. 11 de marzo (ICC-01/04-01/06-2340).

CPI, Sala de Apelaciones. (2015). Prosecutor vs. Thomas Lubanga Dylo. Judgement on the appeals against the 'Decision establishing the principles and procedures to be applied to reparation' of 7 August 2012 with AMENDED order for reparations (Annex A) and public annexes 1 and 2. 3 de marzo (ICC-01/0401/06-3129).

CPI, Sala de Primera Instancia I. (2012a). Prosecutor vs. Thomas Lubanga Dylo. Decision granting leave to make representations in the reparations proceedings. 20 de abril (ICC-01/04-01/06-2870). 
CPI, Sala de Primera Instancia I. (2012b). Prosecutor vs. Thomas Lubanga Dylo. Decision establishing the principles and procedures to be applied to reparations. 7 de agosto (ICC-01/04-01/06-2904).

CPI, Sala de Primera Instancia I. (2012c). Prosecutor vs. Thomas Lubanga Dylo. Judgement pursuant to Article 74 of the Statute. 5 de abril (ICC-01/04-01/06-2842).

CPI, Sala de Apelaciones. (2015). Prosecutor vs. Thomas Lubanga Dylo. Judgement on the appeals against the 'Decision establishing the principles and procedures to be applied to reparation' of 7 August 2012 with AMENDED order for reparations (Annex A) and public annexes 1 and 2. 3 de marzo (ICC-01/04-01/06-3129).

CPI, Sala de Primera Instancia I. (2009). Prosecutor vs. Omar Hassan Amhad Al Bashir. Warrant of Arrest. 4 de marzo (ICC-02/05-01/09-1).

CPI, Sala de Primera Instancia II. (2015a). Prosecutor vs. Ongwen. Decision Establishing Principles on the Victims' Application Process. 4 de marzo (ICC02/04-01/15-205).

CPI, Sala de Primera Instancia II. (2015b). Prosecutor vs. Ongwen. Decision on contested victims' applications for participation, legal representation of victims and their procedural rights. 27 de noviembre (ICC-02/04-01/15-350).

CPI, Sala de Cuestiones Preliminares II. (2011). Prosecutor vs. Ruto and Sang. Decision on Victims 'Participation at the Confirmation of Charges Hearing and in the Related Proceedings. 8 de agosto (ICC-01/09-01/11-249).

CPI, Sala de Cuestiones Preliminares III. (2017). Situation in the Republic of Burundi. Decision Pursuant to Article 15 of the Rome Statute on the Authorization of an Investigation into the Situation in the Republic of Burundi. 25 de octubre (ICC-01/17-X-9-US-Exp).

CPI, Sala de Cuestiones Preliminares III. (2011). Situation in the Republic of Côte d' Ivore. Request for authorisation of an investigation pursuant to article 15.23 de junio (ICC-02/11-3).

CPI, Sala de Cuestiones Preliminares I. (2018). Situation in the State of Palestina. Decision on Inform and Outreach for the Victims of the Situation. 13 de julio (ICC-01/18). 


\section{Otros}

Amnistía Internacional. (2016). Malabo Protocol. Legal and Institutional Implications of the Merged and expanded African Court. Disponible en https:// www.amnesty.org/download/Documents/AFR0130632016ENGLISH.PDF.

Curvellier, T. (2016). The Trial of Hissène Habré. The New York Times. Disponible en https://www.nytimes.com/2016/02/16/opinion/the-landmark-trial-ofhissene-habre.html

Human Rights Watch. (2017). Who Will Stand for Us? Victim's Legal Representation at the ICC in the Ongwen Case and Beyond. Disponible en https:// www.hrw.org/sites/default/files/report_pdf/ijongwen0817_web.pdf 